\title{
Observational characteristics and association of umbral oscillations and running penumbral waves
}

\author{
K. Tziotziou ${ }^{1}$, G. Tsiropoula ${ }^{1}$, N. Mein ${ }^{2}$, and P. Mein ${ }^{2}$ \\ 1 National Observatory of Athens, Institute for Space Applications and Remote Sensing, Lofos Koufos, 15236 Palea Penteli, Greece \\ e-mail: [kostas;georgia] aspace.noa.gr \\ 2 Observatoire de Paris, Section de Meudon, LESIA, 92195 Meudon Principal Cedex, France \\ e-mail: [Nicole.Mein;Pierre.Mein] @obspm.fr \\ Received 10 February 2006 / Accepted 18 April 2006
}

ABSTRACT

\begin{abstract}
Context. Umbral flashes (UFs) and running penumbral (RP) waves are believed to be closely related oscillatory phenomena of sunspots.

Aims. We investigate the association of UFs and RP waves to see whether the latter are a visual pattern created by a common source with UFs or a trans-sunspot wave driven by UFs.

Methods. Simultaneous, two-dimensional, dual-line observations in Ca II $8542 \AA$ and $\mathrm{H} \alpha 6563 \AA$, obtained with the Multichannel Subtractive Double Pass (MSDP) spectrograph mounted on the German VTT at Teide Observatory on Tenerife, are used for this study. High-cadence $8 \mathrm{~s}$ Doppler velocity images, spectrograms, and spectral-analysis results are used to study the characteristics and the relationship of UFs and RP waves.

Results. Several UFs were observed that seem to fill the whole umbra. Doppler velocity variations with time indicate a shock behaviour for UFs, as well as for umbral and RP waves and a smooth continuous propagation of the latter from the umbra through the umbra-penumbra boundary out to the edge of the penumbra. Furthermore, the spectral analysis shows a decreasing oscillatory frequency as we move from the umbra outwards and a jump at the umbra-penumbra boundary that could possibly reflect, apart from a change in physical conditions, a drastic change of the magnetic field inclination with respect to the vertical.

Conclusions. The results do not permit us to convincingly support one scenario over the other (i.e. visual pattern vs. trans-sunspot wave) for RP waves; however, they do provide important constraints for future models of sunspot oscillations and RP waves.
\end{abstract}

Key words. Sun: chromosphere - Sun: oscillations - Sun: sunspots

\section{Introduction}

The study of oscillations in the atmosphere of sunspots began in 1969 with the detection of umbral flashes (UFs) in intensity images of the $\mathrm{Ca}$ II $\mathrm{H}$ and $\mathrm{K}$ lines and of the infrared triplet of Ca II $8542 \AA$ by Beckers \& Tallant (1969). Three years later, intensity and/or velocity in various spectral-line observations revealed the existence of the 5-min oscillations in the umbral photosphere (Bhatnagar et al. 1972) and of the 3-min oscillations in the umbral chromosphere (Beckers \& Schultz 1972; Giovanelli 1972). Chromospheric umbral oscillations are present in almost every sunspot umbra, whereas umbral flashes are localized phenomena that are observed occasionally when the velocity amplitudes exceed a certain value depending on the line. Zirin \& Stein (1972) and Giovanelli (1972) were the first to realize that the penumbra oscillates as well. Using filtergrams obtained in the wings of $\mathrm{H} \alpha$ they detected concentric rings moving from the inner to the outer penumbra; these are called running penumbral (RP) waves.

Since then many observations of RP waves have been reported (see review by Bogdan \& Judge 2006 for references). The RP waves are easily detected in strong chromospheric resonance lines as disturbances propagating from the umbra-penumbra boundary out to the edge of the penumbra and becoming gradually invisible. Their characteristic pattern, observed mainly in velocity images, is the one of alternating dark and bright arcs having radial extents $2300-3800 \mathrm{~km}$, and azimuthal extents of $90^{\circ}-180^{\circ}$ sometimes attaining even $360^{\circ}$. They propagate radially outward with propagation velocities and velocity amplitudes decreasing from the inner to the outer edges of the penumbra (Tsiropoula et al. 2000), while their oscillation period has a peak within the 5-min band (Tziotziou et al. 2002).

The driving source of RP waves and their relationship to umbral oscillations is still a controversial matter. Alissandrakis et al. (1992) detected waves that started in umbral elements and propagated through the penumbra. Tsiropoula et al. $(1996,2000)$ provide clear evidence of waves originating from oscillating elements inside the umbra and propagating through the penumbra. Christopoulou et al. (2000), on the other hand, claim that RP are not the continuation of umbral oscillations into the penumbra, while Rouppe van der Voort et al. (2003) suggest that UFs and RP waves share a common extended source below the visible photosphere. Rouppe van der Voort et al. (2003) also speculate that RP waves are not physical waves, but instead the visual pattern of upward propagating wavefronts along individual magnetic field lines of longer length and greater inclination with respect to the vertical. Recently, Bogdan \& Judge (2006) agree with this idea and present new arguments to support it.

The aim of this work is to study the association of UFs and umbral and RP waves by analysing high cadence observations in two chromospheric lines.

\section{Observations}

A sunspot (NOAA 9641, S13 W19) was observed on October 4, 2001 with the Multichannel Subtractive Double Pass (MSDP) 
spectrograph that is mounted on the German solar Vacuum Tower Telescope (VTT) in Tenerife, Canary Islands. The MSDP spectrograph (Mein 1991, 2002) is designed to record a twodimensional field of view at several wavelengths within a line profile, thus allowing the reconstruction of the line profile at each pixel of the image. Several lines, depending on the configuration of the telescope and the available cameras, can be observed simultaneously. If necessary, a large region of the solar surface can be quickly covered in several wavelengths by displacing the entrance field stop of the telescope.

We have carried out observations of the same field of view simultaneously in two lines, the $\mathrm{H} \alpha$ and the infrared Ca II $8542 \AA$ from 09:53:39 to 10:10:51 UT. Since we were interested in obtaining a high temporal resolution observational sequence, only one two-dimensional $183.5^{\prime \prime} \times 19^{\prime \prime}$ band was recorded (requiring no displacement of the telescope) with a cadence of $8 \mathrm{~s}$ : exposure time of $100 \mathrm{~ms}$ for Ca II $8542 \AA, 20 \mathrm{~ms}$ for $\mathrm{H} \alpha+$ acquisition time of the data. The images were recorded with a relatively good seeing (no correlation tracker used), resulting in a spatial resolution lower than $1^{\prime \prime}$, which is estimated from the visual size of structures at $\mathrm{H} \alpha$ line centre. After correcting for flat field and dark current with the MSDP reduction package, 130 intensity and Doppler velocity images have been obtained for each line. Both were derived using the bisector technique with the mean profile over the field-of-view (avoiding the brightest and darkest regions) as the reference profile. For $\mathrm{H} \alpha$ we obtained two-dimensional intensity images at line-centre, $\pm 0.29 \AA$ and $\pm 0.58 \AA$, and Doppler velocity images at $\pm 0.29 \AA$ and $\pm 0.58 \AA$, while for Ca II $8542 \AA$ we have intensity images at line-centre, $\pm 0.09 \AA$ and $\pm 0.135 \AA$, and Doppler velocity images at $\pm 0.09 \AA$ and $\pm 0.135 \AA$. Positive Doppler shifts correspond to upward Doppler velocities and show up as bright in all presented velocity images. Two-dimensional cross-correlation techniques were used for a careful alignment of the images of our sequence, ensuring the possibility of a direct comparison between pixels in different images of the time series.

\section{Results}

\subsection{Umbral flashes and RP waves}

In Fig. 1 we present a) a Ca II $8542 \AA$ intensity image at $\pm 0.135 \AA$ of the observed sunspot; b) an $\mathrm{H} \alpha$ intensity image at $\pm 0.58 \AA$; c) a Ca II $8542 \AA$ Doppler velocity image at $\pm 0.135 \AA$; d) an $\mathrm{H} \alpha$ Doppler velocity image at $\pm 0.29 \AA$; and e) the distribution of the highest amplitude downward Doppler Ca II $8542 \AA$ velocity at $\pm 0.09 \AA$ over the whole sequence per pixel of the observed field of view. The observed sunspot is isolated and slightly elongated. Its penumbra at the top of the image is, unfortunately, not observed, while at the bottom is only partially observed.

At least five UFs are visible in $\mathrm{H} \alpha$ as high upward or downward Doppler velocities within the umbra, while in Ca II $8542 \AA$ they only show up as high downward Doppler velocities. The latter is the result of both (i) the Ca II $8542 \AA$ UF profile, which shows an emission on its blue side supposedly caused by the upward movement of hot material, and (ii) the derivation of Doppler velocities with the bisector method (Kneer et al. 1981; Uexküll et al. 1983; Tziotziou et al. 2002). The UFs appear in several distinct places within the umbra and seem to fill almost the whole of it, either growing in amplitude and diffusing at the same position or diffusing by slow spreading in their vicinity.

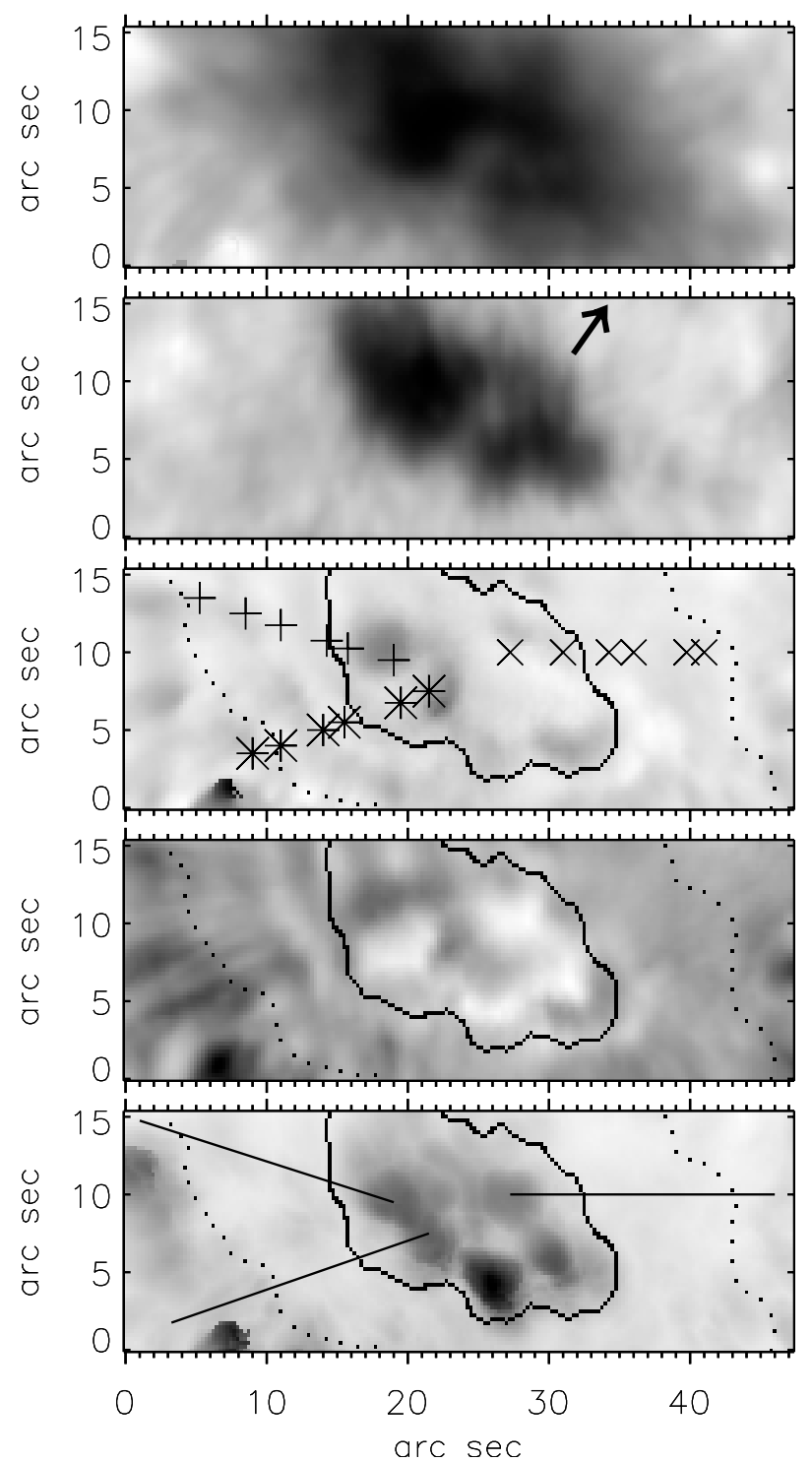

Fig. 1. A Ca II $8542 \AA$ intensity image at $\pm 0.135 \AA$ of the observed sunspot (top panel), an $\mathrm{H} \alpha$ intensity image at $\pm 0.58 \AA$ (second panel), a Ca II $8542 \AA$ Doppler velocity image at $\pm 0.09 \AA$ (third panel), an $\mathrm{H} \alpha$ Doppler velocity image at $\pm 0.29 \AA$ (fourth panel), and the distribution of the highest amplitude downward Doppler velocity of Ca II $8542 \AA$ at $\pm 0.09 \AA$ over the whole sequence per pixel of the observed field of view (bottom panel). In the last three panels, black represents downward velocities. The solid black contour shows the $\mathrm{H} \alpha$ umbra-penumbra boundary, the dotted black contour shows the approximate outer penumbra boundary, overplotted lines indicate the cuts used for the time slice images of Fig. 2, and signs mark the positions along the three cuts of the Doppler velocity profiles of Fig. 3. North is towards the top of the panels, West is towards the left of the panels, while the black arrow in the second panel shows the direction to solar disc centre.

Individual umbral flashes tend to reappear periodically at almost the same location.

The RP waves show up in the Ca II $8542 \AA$ and H $\alpha$ Doppler velocity images as propagating arcs in the penumbra starting at the umbra-penumbra boundary. They are not clearly visible in the whole penumbra but seem to have an azimuthal extent of at least $140^{\circ}$ on the left (southwest) side of the penumbra. In Fig. 2 we present time slice images of $\mathrm{H} \alpha$ Doppler velocity at $\pm 0.29 \AA$ (top row) and Ca II $8542 \AA$ Doppler velocity at 

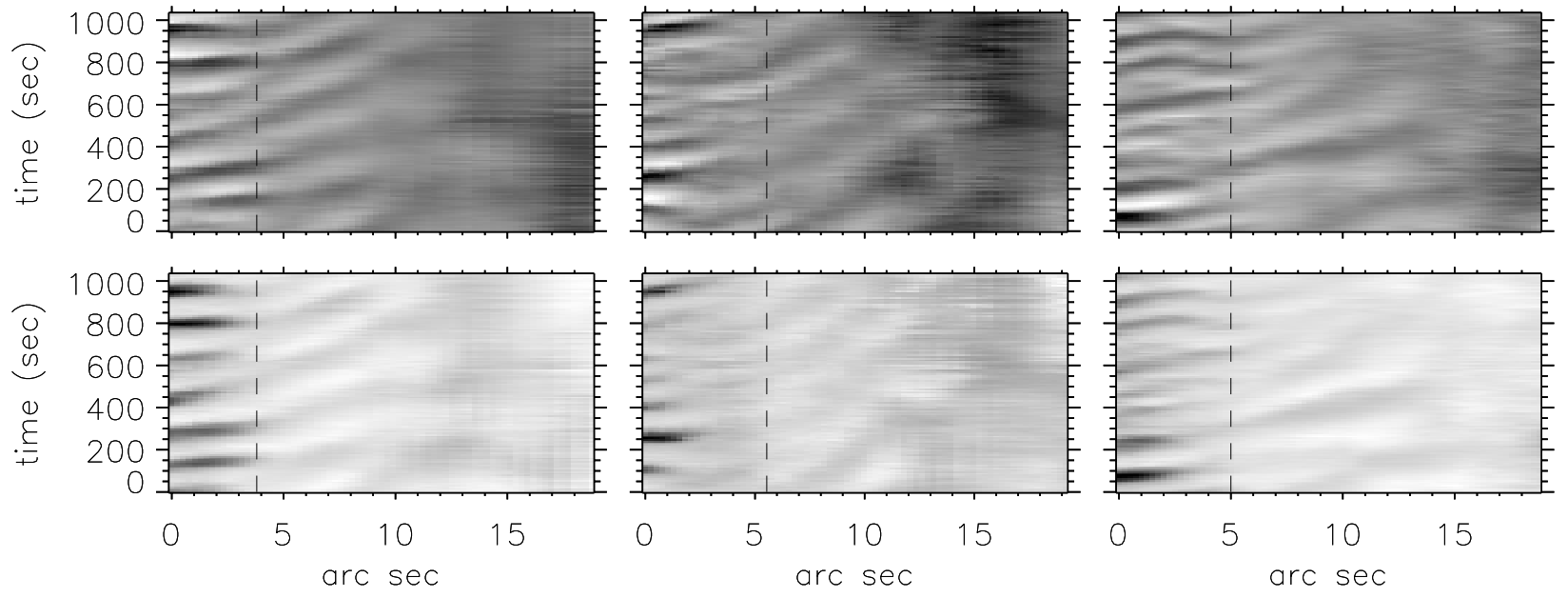

Fig. 2. Time slice images of $\mathrm{H} \alpha$ Doppler velocity at $\pm 0.29 \AA$ (top row) and Ca II $8542 \AA$ Doppler velocity at $\pm 0.135 \AA$ (bottom row) along the top left cut (left column), bottom left cut (middle column), and the right cut (right column) shown in Fig. 1. Black represents downward velocities and length runs from the umbra towards the edge of the penumbra. The vertical dashed line indicates the approximate umbra-penumbra boundary.

$\pm 0.135 \AA$ (bottom row), which is the Doppler velocity as a function of time along the three cuts shown in Fig. 1, starting from an umbral flash position and moving towards the penumbra. There is a characteristic pattern of alternating dark and bright bands, representing downward and upward Doppler velocities respectively. Umbral flashes show up as high-amplitude velocities in the umbra, while the pattern or running penumbra waves is characteristically concave upwards, suggesting a propagation velocity decrease as they propagate from the umbra-penumbra boundary to the outer penumbra. Most of the waves in the penumbra can be traced back inside the umbra and seem to be a continuation of umbral waves, although there are some umbral waves that seem to stop in the umbra-penumbra boundary.

In Fig. 3 we present a series of $\mathrm{H} \alpha$ and Ca II $8542 \AA$ Doppler velocity variations at certain positions along the cuts, presented in Fig. 1 as marked. The top row corresponds to Doppler velocity variations at an umbral flash position. The second row shows Doppler velocity variations at an umbral position just before the umbra-penumbra boundary, while the third and fourth rows show velocity variations for positions within the inner penumbra. Finally the fifth and sixth rows show Doppler velocity variations in the outer penumbra.

One can see that as we move from the umbra towards the edge of the penumbra the Doppler velocity amplitude decreases, while the period of the oscillations increases. The $\mathrm{H} \alpha$ Doppler velocity variations at UFs (top row) show an abrupt increase from negative to positive values followed by a gradual decrease back to negative values. This behaviour is less obvious in the Ca II $8542 \AA$ Doppler velocity curves due to the difficulty of estimating upward velocities for the reasons mentioned above. The sawtooth behaviour of the chromospheric umbral oscillations has already been reported by several authors in the past (see reviews of Lites 1992; Bogdan \& Judge 2006) and is attributed to nonlinear near-acoustic field-guided disturbances formed in the photosphere or below, propagating upward and developing into shocks because of the decreasing density. The interesting thing to notice in the present observations (second to fifth row of Fig. 3) is that the sawtooth behaviour suggestive of shocks is observed not only inside the umbra, but also in the penumbra. This has also been noticed by Rouppe van der Voort et al. (2003), although only in a position close to the umbra-penumbra boundary (see their Fig. 4, last panel). In our case the sawtooth behaviour is seen as far as at the outer edges of the penumbra. It is quite clear in the first three rows but less obvious to identify them in the fourth and fifth rows. However, in these two rows the decreasing part of most wavefronts lasts longer than the rising part, and there are clearly some wavefronts (i.e. 2 nd wavefront in panels of the 3rd row, 2nd wavefront in the 3rd panel of fifth row) where the rising part has a steeper slope than the decreasing part. Only in the last row panels that probably represent positions close to or even in the superpenumbra one is unable to identify any sawtooth profiles.

Figure 4 shows a time series of $\mathrm{H} \alpha$ Doppler velocity at $\pm 0.29 \AA$ (solid line) and Ca II $8542 \AA$ Doppler velocity at $\pm 0.09 \AA$ (dotted line) along the top left cut of Fig. 1 with a time cadence of $8 \mathrm{~s}$. Zero time corresponds to the top left panel and zero length corresponds to an umbral flash position, whose Doppler velocity variability is shown in the top left panel of Fig. 3. We see that the umbral flash (peak at zero length at $t=0 \mathrm{~s}$ ) leads to an umbral wave that propagates outwards at an average speed of $\sim 19 \mathrm{~km} \mathrm{~s}^{-1}$, crossing the umbra penumbra boundary at $t=144 \mathrm{~s}$. Then it continues as a running penumbral wave at an average speed of $\sim 17 \mathrm{~km} \mathrm{~s}^{-1}$ and is visible until the last presented panel at position $\sim 7^{\prime \prime}$ with a much lower velocity amplitude. A new umbral wave starts at $t=184 \mathrm{~s}$ and is close to the umbra-penumbra boundary in the last panel, propagating faster with an average speed of $\sim 25 \mathrm{~km} \mathrm{~s}^{-1}$, but with a more rapid decrease in velocity amplitude. As seen in the figure, the Doppler velocity variation along the cut, as well as its time evolution, is smooth and continuous. Other cuts and time series, not shown here for the sake of space, show exactly the same behaviour.

\subsection{Frequency behaviour from the umbra towards the penumbra}

Figure 5 shows the peak oscillation frequency along cuts starting from the same position in the middle of the umbra $\left(x=26.5^{\prime \prime}\right.$, $y=7.25^{\prime \prime}$ in Fig. 1) and going outwards until they reach the image boundaries, which is why they have a variable length. The cuts go anti-clockwise starting from the cut with an angle of $10^{\circ}$ with respect to the vertical passing from the top and quite a clear 

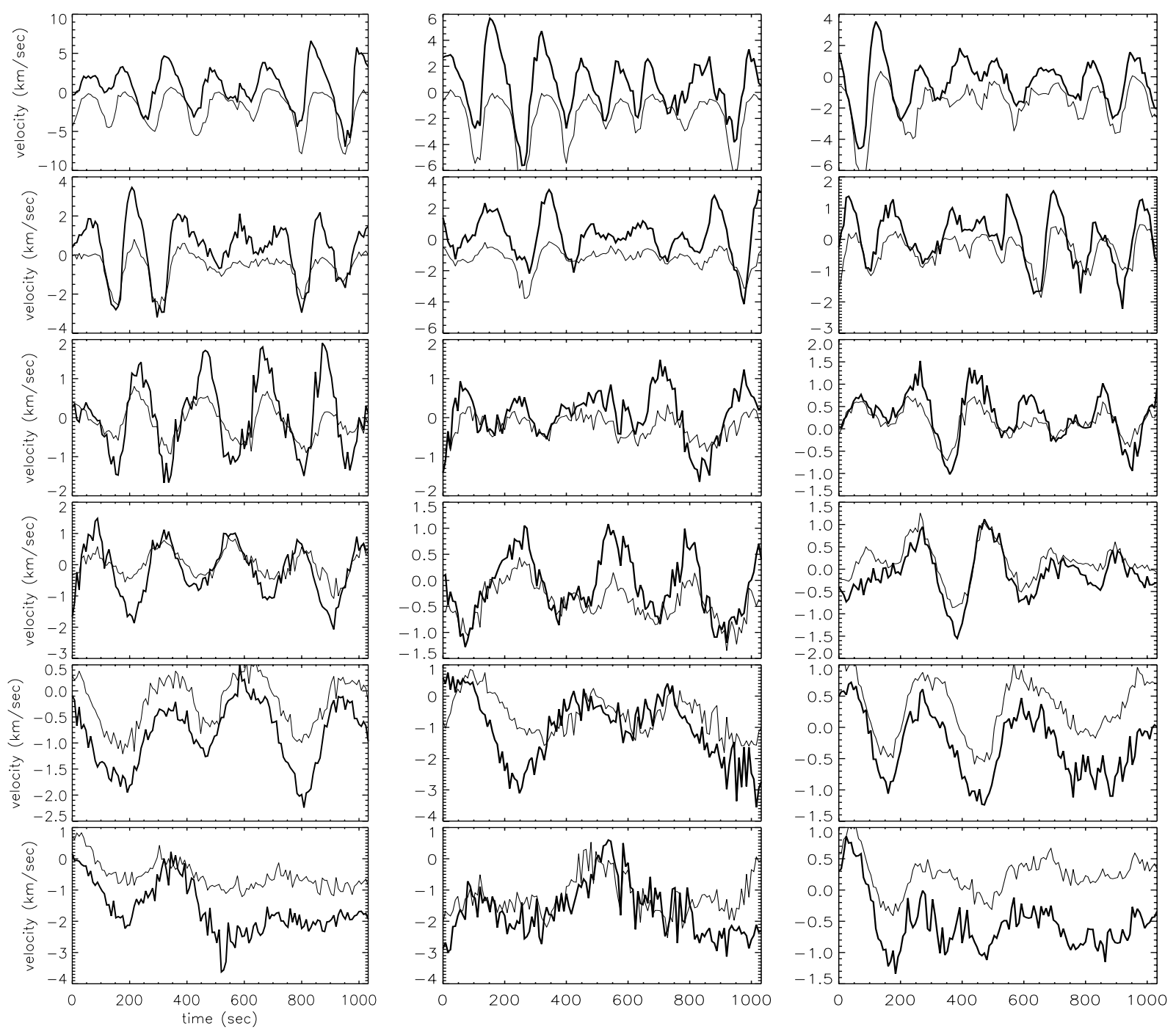

Fig. 3. A series of $\mathrm{H} \alpha$ Doppler velocity variations with time at $\pm 0.29 \AA$ (thick solid line) and Ca II $8542 \AA$ Doppler velocity variations at $\pm 0.09 \AA$ (thin solid line). The first column shows variations at positions marked with the "+" sign in Fig. 1, second column at positions marked with the "*" sign, and the third column at positions marked with the " $\mathrm{x}$ " sign. Doppler velocity variations run from top to bottom from the umbra towards the edge of the penumbra.

oscillatory behaviour in Doppler velocity, like the one shown in Fig. 3, has been observed in all pixels along the used cuts. The peak oscillation frequency was calculated from the $\mathrm{H} \alpha$ Doppler velocity variation at $\pm 0.29 \AA$ and from the Ca II $8542 \AA$ Doppler velocity variation at $\pm 0.09 \AA$ with a wavelet analysis (peak of global power spectrum, see Tziotziou et al. 2004 for details on the method). A two-dimensional frequency analysis in the whole area of the observed sunspot will be presented in a forthcoming paper that is in preparation. The dashed line indicates the approximate umbra-penumbra boundary shown in Fig. 1, which was defined as the isocontour of a specific value of $\mathrm{H} \alpha$ intensity hence should only be considered as indicative. No cuts are shown in Fig. 5 between $10^{\circ}$ and $60^{\circ}$, since for these inclinations we hardly cross the umbra-penumbra boundary due to the limited field of view (as seen in Fig. 1)

We see that the frequency decreases from the umbra outwards, and its behaviour in both lines is almost identical with differences mostly in the umbra rising from the behaviour of the Ca II $8542 \AA$ umbral flash profile as explained in Sect. 3.1. The frequency decrease is not smooth, but there seems to be an abrupt frequency change at the umbra-penumbra boundary; however, a very rapid but smooth transition that is not observed due to our spatial resolution cannot be excluded. This frequency jump probably indicates a drastic change in the local physical conditions - e.g. a substantial increase in the density of the penumbra relative to the umbra - and of the local magnetic field topology.

\subsection{Magnetic field inclination in the umbra and penumbra}

Keppens \& Martínez Pillet (1996) have shown that the inclination of the magnetic field increases rather linearly from about $0^{\circ}$ to the vertical at the centre of the umbra up to $40^{\circ}$ at the umbra-penumbra boundary and continues increasing smoothly outwards, attaining values of $\sim 70^{\circ}-80^{\circ}$ at the outer edge of the sunspot (see also Solanki 2003). In the following we give a qualitative estimation of the inclination of the magnetic field lines using some empirical formulas that relate the inclination angles to the peak frequency of the oscillation spectrum (Bogdan \& Judge 2006, and references cited therein). According to these formulae the predicted peak frequency can be estimated along a 


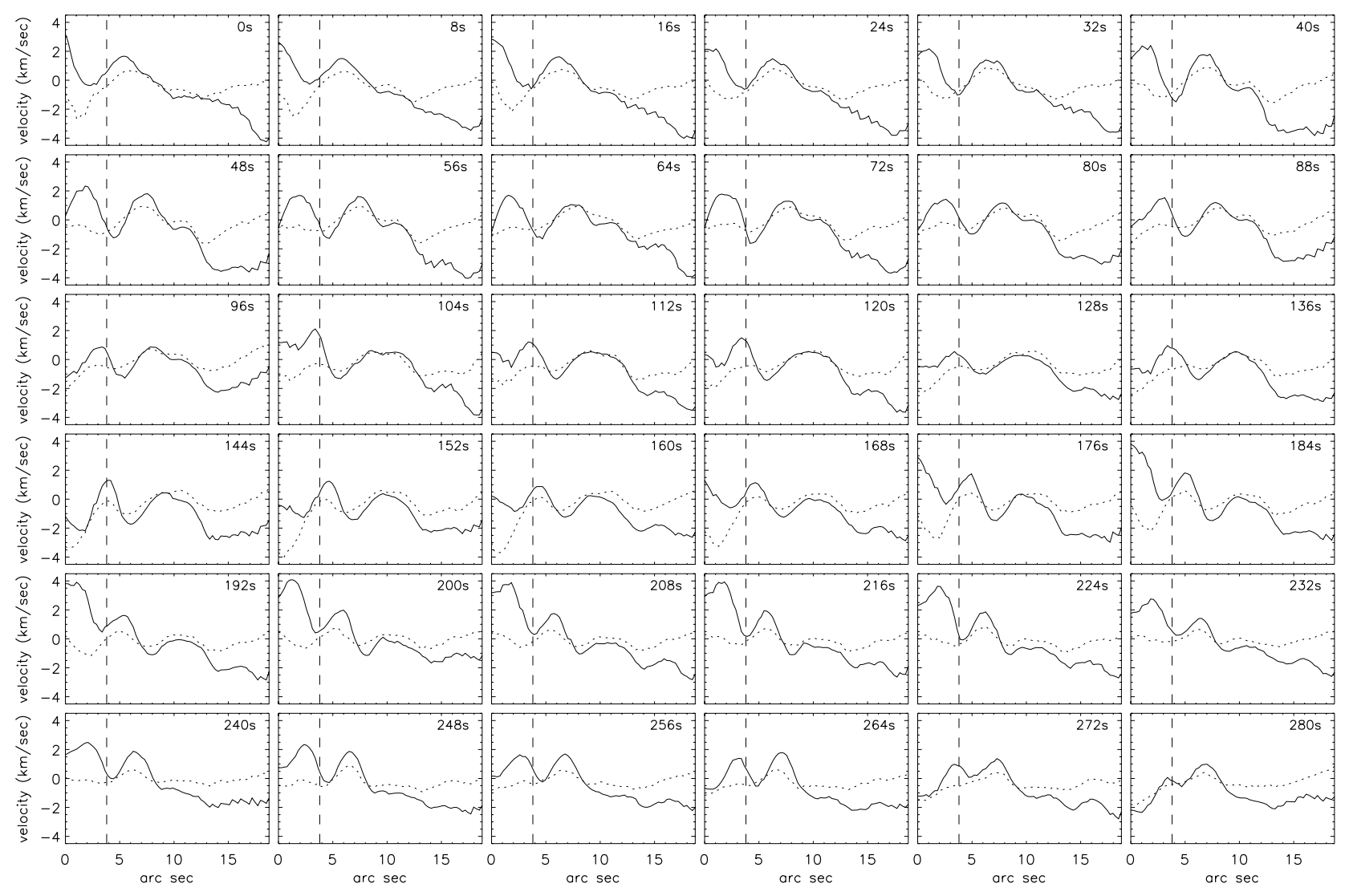

Fig. 4. A time series of $\mathrm{H} \alpha$ Doppler velocity at $\pm 0.29 \AA$ (solid line) and Ca II $8542 \AA$ Doppler velocity at $\pm 0.09 \AA$ (dotted line) along the top left cut of Fig. 1. Zero length corresponds to an umbral flash position, whose Doppler velocity variability is shown in the top left panel of Fig. 3. Time, starting from the top left panel, is indicated within each panel. The time cadence is $8 \mathrm{~s}$. The dashed line indicates the approximate umbra-penumbra boundary.

magnetic field line with inclination $\theta$ from the empirical formula $v_{\text {peak }}(\theta) \approx 1.25 v_{\text {ac }}(\theta)$ where $v_{\text {ac }}$ is the acoustic cut-off frequency that depends on $\theta$ as $v_{\mathrm{ac}}(\theta) \approx 5.2 \cos \theta \mathrm{mHz}$. We stress that these formulas that relate the acoustic cut-off frequency to the magnetic field inclination are valid under very idealized conditions, since they assume that the inclination of the magnetic field lines remains constant with height all the way from the photosphere to the chromosphere. As a result, the derived inclination angles can only be used for a qualitative and not for a quantitative analysis.

Figure 6 shows the respective inclination angles of the magnetic field lines with respect to the vertical calculated with the aforementioned formulas from the $\mathrm{H} \alpha$ Doppler velocity variations at $\pm 0.29 \AA$ and from the Ca II $8542 \AA$ Doppler velocity variations at $\pm 0.09 \AA$ along the cuts of Fig. 5. As can be seen from the empirical formulas, no angle can be derived for peak frequencies higher than $6.5 \mathrm{mHz}$, which explains the gaps in the figure and is another indication of the limitations of these formulas. It is also obvious that the inclination angles are lower inside the umbra (fields closer to vertical) and attain higher values (more horizontal fields) after crossing the umbra-penumbra boundary. For most of the cuts there are no significant changes in the magnetic field inclination within the umbra. This contradicts the general view based on spectropolarimetric measurements that the magnetic field inclination increases smoothly outwards in the observed sunspot. If it is indeed real, it could probably reflect the fact that UFs appear localized but quite extended, suggesting that they are wavefronts travelling along magnetic field lines of similar inclination over a large area. The origin of the contradiction between spectropolarimetric measurements and these results is not yet clear. It could be attributed to the magnetic field inclination being estimated by different analyses at different heights in the photospheric and/or chromospheric atmosphere where the magnetic field topology may be different; however, further work is necessary to confirm or disprove such a hypothesis, not excluding of course other potential solutions to this problem. The inclination angles at the umbra-penumbra boundary show a jump, reflecting the jump in frequencies, which is normal, since the values of these latter are used for their estimation. This jump is highly questionable, since the formulas cannot be used to obtain precise values as stressed above. It would, however, if real, indicate a drastic change of the magnetic field topology at the umbra-penumbra boundary.

\section{Discussion and conclusions}

High temporal cadence observations in Ca II $8542 \AA$ and $\mathrm{H} \alpha$ have been analyzed to provide additional information about UFs, RP waves, and their possible association. The analysis reveals a large number of extended umbral flashes that, taken together, seem to fill almost the whole umbra at any given moment. RP waves can be traced inside the umbra and most of them seem visually to be a continuation of umbral flashes and umbral waves. They are propagating outwards with decreasing radial speed, and velocity variations in the penumbra show a sawtooth shape similar to the sawtooth behaviour of UFs, although they are less sharp, implying that RP waves also possess similar shock-like behaviour along the line-of-sight. 

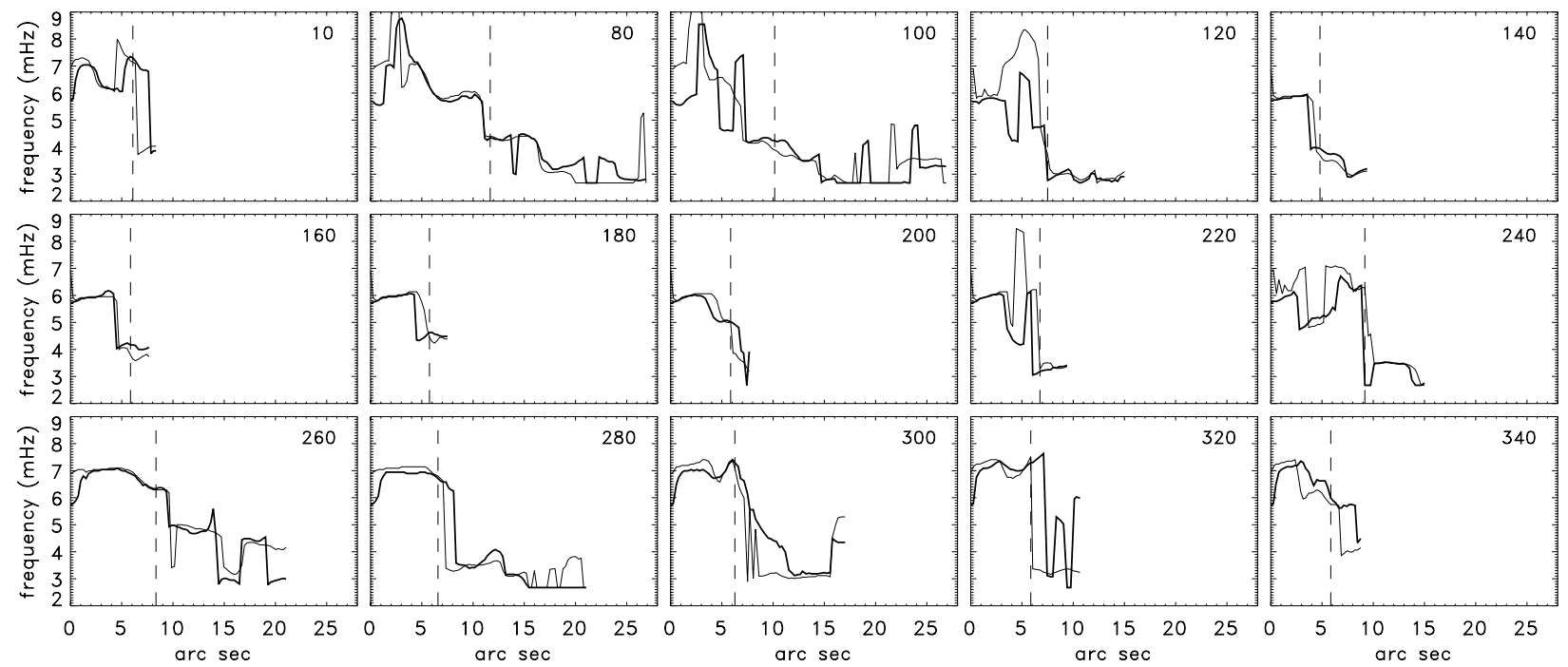

Fig. 5. Peak frequency of oscillation calculated from the $\mathrm{H} \alpha$ Doppler velocity variations at $\pm 0.29 \AA$ (thick solid line) and from the Ca II $8542 \AA$ Doppler velocity variations at $\pm 0.09 \AA$ (thin solid line) along cuts starting from the same position in the middle of the umbra $\left(x=26.5^{\prime \prime}, y=7.25^{\prime \prime}\right.$ in Fig. 1) and going outwards until they reach the image boundaries. The number in the top right corner indicates the anti-clockwise angle of the cut with respect to the top vertical. The dashed line indicates the approximate umbra-penumbra boundary.

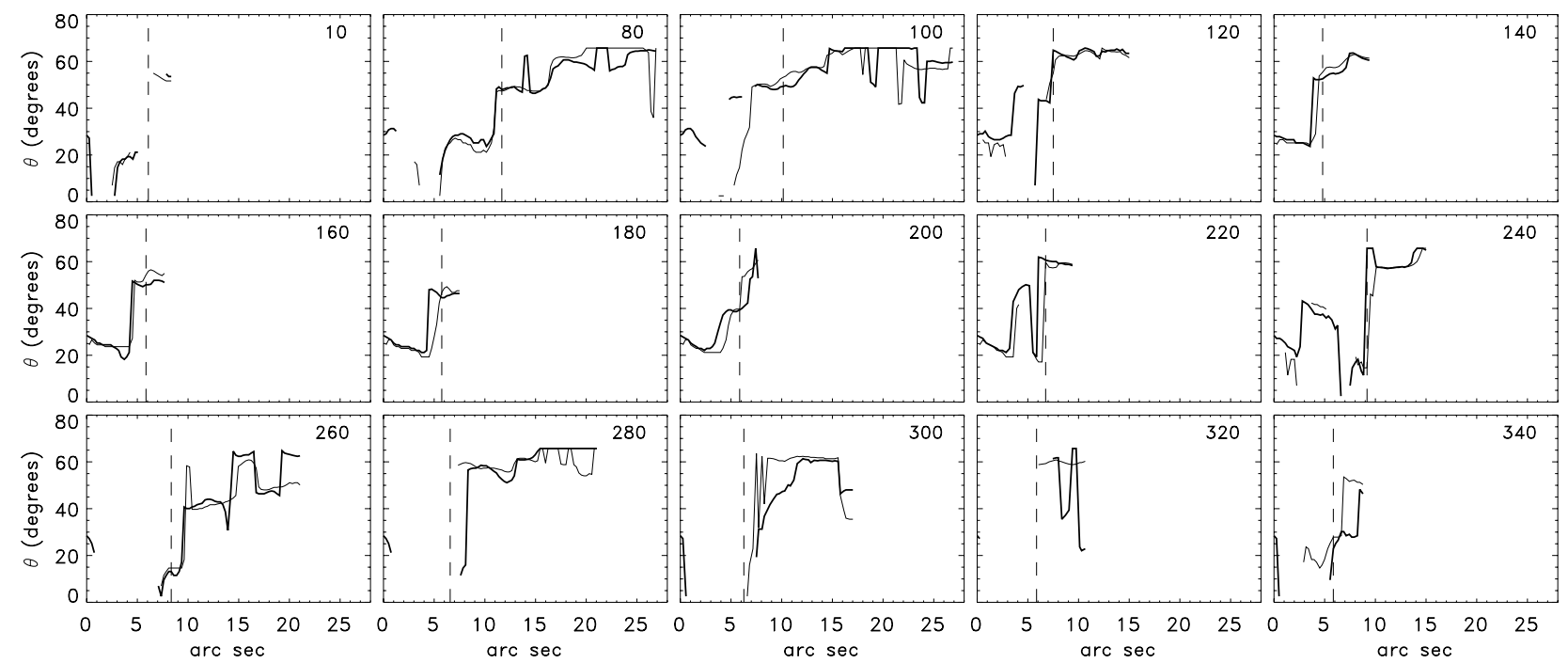

Fig. 6. Inclination angle $\theta$ of the magnetic field lines calculated from the $\mathrm{H} \alpha$ Doppler velocity variations at $\pm 0.29 \AA$ (thick solid line) and from the Ca II $8542 \AA$ A Doppler velocity variations at $\pm 0.09 \AA$ (thin solid line) along the cuts of Fig. 5 . The number in the top right corner indicates the anti-clockwise angle of the cut with respect to the top vertical. The dashed line indicates the approximate umbra-penumbra boundary.

An important observational finding of our analysis is the substantial decrease in oscillation frequency at the umbra-penumbra boundary. Due to our spatial resolution, it is not absolutely clear if it is an abrupt jump in the frequency or a smooth but rapid transition. Such a decrease was demonstrated before by Tziotziou et al. (2002), although with lower temporal cadence observations. In the present observations this decrease is quite clear along the whole umbra-penumbra boundary and is found in frequencies derived both from Ca II $8542 \AA$ and $\mathrm{H} \alpha$ Doppler velocity variations.

Nowadays umbral and RP waves are believed to be either a) a visual pattern of a later wavefront arrival of the same disturbance that creates UFs, due to a longer travel path along divergent sunspot field lines, or b) a physical, trans-sunspot wave travelling from the umbra outwards (see introduction for references). In the first case, UFs, umbral, and running penumbral waves are believed to be sharing a common extended source region somewhere below the visible photosphere, and in the latter case UFs are believed to be the drivers of umbral and RP waves. There are a number of observational features that a realistic sunspot scheme should be able to explain. These are: a) the frequency changes, b) the decreasing radial propagation speed of RP waves, c) the sawtooth velocity profiles observed well inside the penumbra, d) the smooth wave propagation from the umbra to the penumbra shown in Fig. 4, and e) the observed frequency jump at the umbra-penumbra boundary.

Frequency and propagation speed changes are naturally explained in the visual pattern scenario and are attributed to longer propagation of wavefronts along diverging individual magnetic field lines as we move from the umbra to the penumbra 
(Bogdan \& Judge 2006). In the physical trans-sunspot wave scenario, the substantial frequency and radial phase speed decrease as it travels from the umbra to the penumbra could also be explained as due to changes in local physical conditions and in the magnetic field topology, as well as to the inverse Evershed flow, since the waves have to propagate against the flow. Dere et al. (1990), Tsiropoula et al. (1996), and Georgakilas et al. (2003) find Evershed radial inflow velocities at $\mathrm{H} \alpha \pm 0.3 \AA$ of the order of $2.6 \mathrm{~km} \mathrm{~s}^{-1}, 4 \mathrm{~km} \mathrm{~s}^{-1}$, and $6 \mathrm{~km} \mathrm{~s}^{-1}$, respectively. The used computational method is based on integration over wide angles in order to compute the average flow velocity vector that is generally believed to be underestimated. It is thus not easy to estimate how significant the Evershed inflow is on the frequency and propagation speed changes.

The sawtooth velocity profiles in the penumbra could be explained by both schemes. In the visual pattern scenario, the origin of such a sawtooth profile would be the same as that of umbral flashes: propagating waves steepening into shocks due to the strong density reduction, a mechanism that should be independent of the inclination of the magnetic field lines and thus work equally well in the umbra and the penumbra. In the transsunspot wave scenario, if umbral and RP waves are indeed a physical wave driven by umbral flashes then one would expect that they would keep the shock signature of their driver as they propagate outwards.

The smooth propagation from the umbra to the penumbra shown in Fig. 4 is an obvious natural consequence in the case of the trans-sunspot wave scenario. The visual pattern scenario would also have no trouble explaining such a smooth propagation across the umbra-penumbra boundary as due to relative travel time differences of upward propagating waves along individual field lines of different inclinations, provided that the magnetic field varies smoothly from the umbra to the penumbra. Spectropolarimetric observations (Keppens \& Martínez Pillet 1996; Solanki 2003) show that the inclination of the magnetic field varies smoothly within the umbra reaching up to $40^{\circ}$ at the umbra-penumbra boundary. However, our observations suggest that this might not be true in our case. When UFs appear they are quite extended, suggesting that they are wavefronts travelling along magnetic field lines of similar inclination over a large area. This is further supported by the magnetic field inclination analysis of Sect. 3.3, which although it cannot be used to derive precise values of the magnetic field inclination - for reasons explained therein - it can give a qualitative picture. The same analysis also suggests that in most parts of the umbra the magnetic field inclination does not vary significantly. If this is indeed the case, then the visual pattern scenario would not be able to explain the smooth propagation observed in Fig. 4.

The frequency jump observed at the umbra-penumbra boundary cannot be easily explained by any of the two scenarios. It probably indicates a drastic change in the local physical conditions, such as a substantial increase in the density of the penumbra relative to the umbra, and/or in the local magnetic field topology from almost vertical to more horizontal. If the latter is true, then the visual pattern scenario would fail to explain the smooth propagation across the umbra-penumbra boundary, while it is not clear how it would be explained by the transsunspot wave scenario.

In conclusion, the idea of umbral and RP waves being a visual pattern of the same disturbance that causes UFs has many attractive features, since it explains frequency and propagation speed changes from the umbra outwards, the sawtooth shape of velocity profiles in the penumbra and - provided that the magnetic field inclination varies smoothly - the smooth propagation outwards. On the other hand, the trans-sunspot waves driven by UFs could explain the penumbral sawtooth profiles and the smooth propagation outwards, as well as frequency and propagation speed changes that can probably be attributed to changes in the local physical conditions and to the influence of the inverse Evershed flow. The frequency jump is not obvious for any of the two scenarios. However, in conjunction with the observed smooth propagation of Fig. 4, it provides important constraints for any realistic sunspot oscillation simulations and theoretical modelling of umbral oscillations and RP waves.

Acknowledgements. The VTT telescope is operated on the island of Tenerife by the Kiepenheuer Insitute in the Spanish Observatorio del Teide of the Instituto de Astrofísica de Canarias. We would like to thank the anonymous referee for comments and suggestions that helped to improve the paper. K.T. acknowledges support by the Marie Curie European Reintegration Grant MERG-CT-2004-021626.

\section{References}

Alissandrakis, C. E., Georgakilas, A. A., \& Dialetis, D. 1992, Sol. Phys., 138, 93 Beckers, J. M., \& Tallant, P. E. 1969, Sol. Phys., 7, 351

Beckers, J. M., \& Schultz, R. B. 1972, Sol. Phys., 27, 61

Bhatnagar, A., Livingston, W. C., \& Harvey, J. W. 1972, Sol. Phys., 27, 80

Bogdan, T. J., \& Judge, P. G. 2006, Phil. Trans. R. Soc. A, 364, 313

Christopoulou, E. B., Georgakilas, A. A., \& Koutchmy, S. 2000, A\&A, 354, 305

Dere, K. P., Schmieder, B., \& Alissandrakis, C. E. 1990, A\&A, 233, 207

Georgakilas, A. A., Christopoulou, E. B., Skodras, A., \& Koutchmy, S. 2003, A\&A, 403, 1123

Giovanelli, R. G. 1972, Sol. Phys., 27, 71

Keppens, R., \& Martínez Pillet, V. 1996, A\&A, 316, 229

Kneer, F., Mattig, W., \& Uexküll, M. v. 1981, A\&A, 102, 147

Lites, B. W. 1992, in NATO ASIC Proc. 375, Sunspots: Theory and Observations, ed. J. H. Thomas, \& N. O. Weiss (Dordrecht: Kluwer Academic), 261

Mein, P. 1991, A\&A, 248, 669

Mein, P. 2002, A\&A, 381, 271

Rouppe van der Voort, L. H. M., Rutten, R. J., Sütterlin, P., Sloover, P. J., \& Krijger, J. M. 2003, A\&A, 403, 277

Solanki, S. K. 2003, A\&AR, 11, 153

Tsiropoula, G., Alissandrakis, C. E., \& Dialetis, D., \& Mein, P. 1996, Sol. Phys., 167,79

Tsiropoula, G., Alissandrakis, C. E., \& Mein, P. 2000, A\&A, 355, 375

Tziotziou, K., Tsiropoula, G., \& Mein, P. 2002, A\&A, 381, 279

Tziotziou, K., Tsiropoula, G., \& Mein, P. 2004, A\&A, 423, 1133

Uexküll, M. V., Kneer, F., \& Mattig, W. 1983, A\&A, 123, 263

Zirin, H., \& Stein, A. 1972, ApJ, 178, L85 